\title{
A systematic review of the responsiveness of lower limb physical performance measures in inpatient care after stroke
}

\author{
Katharine Scrivener ${ }^{1,2^{*}}$, Catherine Sherrington ${ }^{2}$ and Karl Schurr ${ }^{1}$
}

\begin{abstract}
Background: Responsiveness refers to a measurement tool's ability to detect change in performance over time. The aim of the review was to summarise studies of responsiveness of lower limb physical performance measures during inpatient care after stroke.

Methods: A systematic literature review was conducted. Prospective studies that included participants with a diagnosis of stroke, were commenced in the acute or subacute phase of inpatient care and included a measure of a lower limb physical performance were included in this review.

Results: Twenty-one studies met these inclusion criteria. A variety of measures were investigated including the Berg Balance Scale, various timed walking tests and the Rivermead Mobility Index. Ten of the included studies had small sample sizes (50 participants or less), 2 studies used a convenience sample rather than consecutive recruitment and 5 studies excluded potential participants with poor physical abilities at baseline. Responsiveness varied between and within studies but was generally large, Effect Size (ES) or Standardised Response Mean $(S R M)>0.8$. Measures displaying large responsiveness included the twelve-minute walk test (SRM 1.90) and the Modified Rivermead Mobility Index (SRM 1.31) when re-measured at four weeks after stroke, and the Berg Balance Scale (ES 1.11) and Postural Assessment Scale for Stroke Patients (ES 1.12) when re-measured at approximately six months after stroke.

Conclusion: Studies conducted to date have generally found physical performance measures after stroke to have large responsiveness i.e., to be able to detect changes. Further investigation of the responsiveness of measurement tools after stroke in larger prospective cohort studies is required.
\end{abstract}

Keywords: Stroke, Responsiveness, Walking, Mobility, Measurement, Rehabilitation

\section{Background}

Rehabilitation after stroke aims to optimise stroke survivors' physical functioning including mobility. Physical performance measures involve measurement of mobility and other functional activities. These measures are often used to monitor progress in clinical settings and used in research studies to evaluate the effectiveness of rehabilitation programs. Many different measurement tools are available to evaluate physical performance in stroke survivors [1]. Clinicians and researchers need to decide on

\footnotetext{
* Correspondence: katharine.scrivener@sswahs.nsw.gov.au

'Bankstown-Lidcombe Hospital, 70 Eldridge Rd, Bankstown, NSW 2200, Australia

${ }^{2}$ The George Institute for Global Health, The University of Sydney, PO Box M201Missenden Road, Sydney, NSW 2050, Australia
}

the best measurement tools to use after stroke. In order to make this decision each measurement tool's reliability, validity and responsiveness must be considered [2,3].

Responsiveness is a measurement tool's ability to detect change in performance over time [4]. If responsiveness of a measurement tool is low then even though a person's physical performance may be improving the tool may not be sensitive enough to detect this change e.g. his/her score on a particular scale may not change. Different measurement tools may be more sensitive to change in different settings over different timeframes.

To date, responsiveness has not received the same attention in the literature as reliability and validity [5]. Despite this there has been a recent increase in research

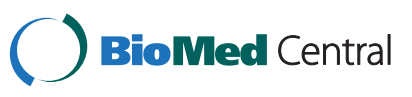


about the responsiveness of measures in stroke rehabilitation. This interest has lead to a number of papers being published summarising the responsiveness of one or more measurement tools. The aim of this systematic review was to summarise the current evidence about the responsiveness of lower limb physical performance measures during inpatient care after stroke. The purpose of this review is to provide clinicians and researchers access to a summary of the many measurement tools available and to assist their selection of the most relevant measurement tool.

The specific research question of this review was: how responsive are measurement tools that measure any aspect of lower limb physical performance in stroke survivors when the use of the measure commences in inpatient care, that is early after stroke?.

\section{Method}

When designing and conducting this systematic review we followed the PRISMA guidelines for systematic reviews [6].

\section{Search strategy}

A literature search was completed of Medline (via OvidSP, 1950 to April 2012), CINAHL (1981 to April 2012) and EMBASE (1980 to April 2012) databases for relevant articles. The search terms were stroke (or cerebrovascular accident or hemiplegia) and responsiveness and terms related to lower limb activities or physical therapy (including sitting or standing or standing up or sit to stand or balance or walking or gait or mobility or physiotherapy or physical therapy or rehabilitation). The first author reviewed titles and/or abstracts of displayed articles and determined relevance to the review. Full text copies of relevant articles were obtained. Reference lists were screened for identification of other relevant articles. Two known systematic reviews of measurement in neurological populations were screened for relevant articles [5,7]. Only articles written in English were included in this review. Any year of publication was included as restricted by each database's availability.

\section{Inclusion criteria}

Studies were included in the review if they: included only participants with a diagnosis of stroke; used a prospective design which involved initial measurement in inpatient care (acute hospital or subacute rehabilitation setting); involved a physical performance measure that related to a lower limb activity including seated reach, standing up, standing, balancing and walking.

A physical performance measure was defined as any measure that required the stroke survivor to actively move or participate. Each measure included use of the legs e.g. sitting, seated reach, moving from sitting to standing, balancing in standing and walking. If the first author was unfamiliar with a scale, a full copy of the scale was reviewed to check for inclusion of lower limb activities. Where upper limb activities were combined in the scales total score the scale was excluded from the review.

\section{Data extraction}

Information about the study design, setting, participants and results were extracted by the first author and checked by the third author. Authors were contacted where there was information missing. When extracting data we looked for an Effect Size I (ESI) (also known as Cohen's Effect Size, the difference between the mean baseline and follow-up scores divided by the standard deviation of baseline scores) or a Standardised Response Mean (SRM) (also known as Effect Size II, the ratio of observed change and its standard deviation, which therefore reflects the variability of change). However if an ESI or SRM was not calculated then, where possible, we calculated one from available data. We considered Effect Size/SRMs of 0.20 to 0.50 be small, 0.50 to 0.80 to be moderate and $>0.8$ to indicate be large [4]. A recent consensus statement indicated that any statistical measure of change, including looking at the difference between before and after measures could be used to measure responsiveness [8]. Consequently if any statistical measure of change was used the article was included in this review.

\section{Assessing risk of bias}

The risk of bias was evaluated for each study using the recent consensus statement regarding design of responsiveness studies as a guideline [8]. In particular we examined each studies sample size and the method used to select participants.

\section{Results}

\section{Flow of studies through the review}

The electronic search identified 189 articles. After screening all titles and abstracts, 26 articles were identified but after reviewing the full text, five of these were excluded from the review. The main reasons for exclusion was that the measurement tool involved an upper limb component or the population sample included people with a diagnosis other than stroke. A systematic review of the Berg Balance Scale was identified in the search and its reference list was also screened, identifying one extra article that had not been found in the electronic search $[9,10]$. When the reference lists of the two previously identified systematic reviews were screened, one additional article was identified and included in the review [11].

Upon reviewing the reference lists and abstracts of potentially relevant papers, from the 21 articles identified by this search, no extra articles were found to be relevant. 


\section{Characteristics of included studies}

The 21 included studies involved 1,101 participants (some of the included studies reported data for the same participants, where this was the case the participant was counted only once). A variety of measurement tools captured a number of lower limb skills ranging from seated reach to standing balance and walking. A summary of the studies is presented in Table 1. Additional information was obtained from the author of one study [12]. An Effect Size was calculated in one study where data was available but an Effect Size was not included in the original article [13].

Most of the included studies were prospective cohort studies. Limitations of studies include ten out of the 21 studies having small sample sizes, 16 to 50 participants $[10,13,14,16,21-23,26,27,29]$, use of a sample of convenience not consecutive stroke survivors $[15,23]$ and having significant exclusion criteria e.g. excluding stroke survivors based on their physical abilities on admission $[11,14,16,22,30]$. Six studies in the review investigated the responsiveness of different measures in the same cohort of stroke survivors; in this case data were collected as part of a larger prospective study [17,19,20,27,30,31].

\section{Time after stroke}

The time post stroke varied across the studies from 7 days to 2 months. Some studies included participants on admission to inpatient rehabilitation. In many of the studies occurring in subacute rehabilitation, the exact time post stroke onset was not indicated $[11,16,22,26,28]$.

\section{Timeframe of follow up}

The time between initial measurement and follow up varied greatly, from one to two weeks to 360 days after the initial measurement. A number of studies followed participants from admission until discharge from hospital [11-13,26,29]; consequently the exact timing between measures was unknown.

\section{Setting}

All participants' measurements commenced early in an inpatient hospital admission. Eleven of the studies specified they were conducted in inpatient rehabilitation. Three were conducted on acute hospital wards. The remaining seven studies indicated that they were conducted in an inpatient hospital setting but further details were not provided.

\section{Outcome measures}

The measurement tools investigated varied. The Berg Balance Scale (BBS) was most investigated, with five studies included in this review investigating the full scale and two investigating a shortened version. For further details of measures investigated refer to Table 1 .

\section{Responsiveness of measures of lower limb physical performance \\ Seated reach}

The Modified Functional Reach Test towards the paretic side showed good responsiveness (ES 0.80) [14]. This reach direction was more responsive than the reach forward or towards the non-paretic side (ES 0.60 and 0.57 respectively).

\section{Balance}

The Berg Balance Scale demonstrated varied responsiveness depending on the study and time of measure post stroke (ES $0.40-1.11$, ES > 1 in 3/9 measures and SRM $0.67-1.29, \mathrm{SRM}>1$ in $2 / 4$ measures) [11,16-19]. A three item short form of the BBS was shown to have similar results (SRM 0.70 and 1.11) [19].

\section{Walking}

The two-minute walk test, 2MWT (SRM 1.34) [23], sixminute walk test, 6MWT (SRM 1.52) [23] and twelveminute walk test, 12MWT (SRM 1.90) [23] were highly responsive to change. However, they were investigated in a small study of only 18 participants. The $5 \mathrm{~m}$ (ES 0.660.81 and SRM $1.00-1.22>1$ in $2 / 2$ measures) $[11,16]$ and $10 \mathrm{~m}$ (ES $0.55-1.17$, ES > 1 in $1 / 3$ measures and SRM 0.83-0.92) [16,22] walk tests demonstrated variable responsiveness.

\section{Multiple}

A number of studies investigated tools that rated subjects based on their ability to complete a number of activities from sitting to walking. Highly responsive measures included the Rivermead Mobility Index (ES 0.891.28 , ES > 1 in $1 / 2$ measures and SRM 0.24-1.14, SRM > 1 in $1 / 3$ measures) $[13,27,28]$, the Modified Rivermead Mobility Index (ES 1.15 and SRM 0.20-1.31, SRM > 1 in $1 / 3$ measures) $[27,29,32]$ and the Modified Emory Functional Ambulation Profile (SRM 1.1) [26]. The lower limb items of the Motor Assessment Scale (MAS) varied in their responsiveness (ES 0.61-1.03, ES > 1 in $2 / 5$ items) [11]. The most responsive items were item 1 ; supine to side lying (ES 1.03) and item 5; walking (ES 1.02) [11]. The Postural Assessment Scale for Stroke patients (PASS) demonstrated variable responsiveness (ES 0.311.12 , ES > 1 in 2/6 measures and SRM 0.84-1.02, SRM > 1 in $1 / 3$ measures) $[17,19,21,33]$. It was most responsive when measured at later time periods after stroke e.g. comparing measures taken at 90 and 180 days post stroke (SRM 1.02) [19] and comparing measures taken at 14 and 180 days post stroke (ES 1.12) [17]. Full details of the responsiveness of each measurement tool, can be seen in Table 1. 
Table 1 Summary of studies investigating the responsiveness of physical performance measures during inpatient care after stroke, $\mathbf{n}=\mathbf{2 1}$

\begin{tabular}{|c|c|c|c|c|c|c|}
\hline Activity & Measure & Study & Setting & $\begin{array}{l}\text { Inclusion timeframe post- } \\
\text { stroke }\end{array}$ & $\mathbf{n}$ & $\begin{array}{l}\text { Timeframe of } \\
\text { reassessment, Result }\end{array}$ \\
\hline \multirow{4}{*}{$\begin{array}{l}\text { Seated } \\
\text { Reach }\end{array}$} & \multirow{4}{*}{$\begin{array}{l}\text { Modified } \\
\text { Functional } \\
\text { Reach Test }\end{array}$} & \multirow{4}{*}{$\begin{array}{l}\text { Katz-Leurer } \\
2009 \text { [14] }\end{array}$} & \multirow{4}{*}{$\begin{array}{l}\text { Inpatient } \\
\text { rehabilitation }\end{array}$} & \multirow[t]{4}{*}{ 14-21 days post stroke } & \multirow[t]{4}{*}{$\mathrm{n}=35$} & 6 wks, Reach direction: \\
\hline & & & & & & Paretic side, $\mathrm{ES}=0.80$ \\
\hline & & & & & & Forward, ES $=0.60$ \\
\hline & & & & & & Non-paretic side, ES $=0.57$ \\
\hline \multirow[t]{33}{*}{ Balance } & \multirow[t]{3}{*}{ PCBS } & \multirow{3}{*}{$\begin{array}{l}\text { Pyoria } \\
2005[15]\end{array}$} & \multirow{3}{*}{$\begin{array}{l}\text { Acute neurological } \\
\text { ward }\end{array}$} & \multirow[t]{3}{*}{7 days post stroke } & $n=50$ & $7-120$ days, $p<0.001$ \\
\hline & & & & & $n=42$ & $120-360$ days, $p>0.05$ \\
\hline & & & & & $n=42$ & $7-360, p<0.001$ \\
\hline & \multirow[t]{11}{*}{ BBS } & $\begin{array}{l}\text { Salbach } \\
2001[16]\end{array}$ & Acute care hospital & $<8$ days (as soon as ambulatory) & $\mathrm{n}=50$ & 4 wks, SRM $=1.04$ \\
\hline & & $\begin{array}{l}\text { Stevenson } \\
2001[10]\end{array}$ & $\begin{array}{l}\text { Stroke Unit } \\
\text { (rehabilitation) }\end{array}$ & $\begin{array}{l}\text { Admission, mean } 30.3 \text { days } \\
\text { (SD 23.3) }\end{array}$ & $n=45$ & $1-2 w k s, p<0.001$ \\
\hline & & \multirow{5}{*}{$\begin{array}{l}\text { Mao } \\
2002[17]\end{array}$} & \multirow[t]{5}{*}{ Hospital inpatients } & \multirow[t]{5}{*}{14 days post stroke } & $n=110$ & 14 to 30 days, $E S=0.80$ \\
\hline & & & & & $\mathrm{n}=93$ & 30 to 90 days, $\mathrm{ES}=0.69$ \\
\hline & & & & & $\mathrm{n}=80$ & 90 to 180 days, $E S=0.40$ \\
\hline & & & & & $n=93$ & 14 to 90 days, $E S=1.07$ \\
\hline & & & & & $\mathrm{n}=80$ & 14 to 180 days, ES = 1.11 \\
\hline & & $\begin{array}{l}\text { English } \\
2006[11]\end{array}$ & $\begin{array}{l}\text { Inpatient } \\
\text { rehabilitation }\end{array}$ & Within 1 wk of admission & $n=61$ & $\begin{array}{l}\text { Within } 1 \text { wk of discharge } \\
\text { (mean time admission to } \\
\text { discharge } 56.4+/-38.1 \text { days), } \\
\text { ES }=1.01\end{array}$ \\
\hline & & \multirow{3}{*}{$\begin{array}{l}\text { Wood- } \\
\text { Dauphinee } \\
1996[18]\end{array}$} & \multirow[t]{3}{*}{$\begin{array}{l}\text { Acute hospital } \\
\text { inpatients }\end{array}$} & \multirow[t]{3}{*}{$<2$ wks post stroke } & \multirow[t]{3}{*}{$n=60$} & $\begin{array}{l}\text { 2wks to } 6 w k s, E S=0.66 \\
S R M=0.81\end{array}$ \\
\hline & & & & & & $\begin{array}{l}\text { 6wks to } 12 w k s, E S=0.25 \\
S R M=0.69\end{array}$ \\
\hline & & & & & & $\begin{array}{l}\text { 2wks to } 12 w k s, E S=0.97 \\
S R M=1.08\end{array}$ \\
\hline & \multirow[t]{3}{*}{ BBS-3P } & \multirow{3}{*}{$\begin{array}{l}\text { Wang } \\
2004[19]\end{array}$} & \multirow[t]{3}{*}{ Hospital inpatients } & \multirow[t]{3}{*}{14 days post stroke } & $n=202$ & 14 to 30 days, SRM $=0.82$ \\
\hline & & & & & $\mathrm{n}=167$ & 30 to 90 days, SRM $=0.70$ \\
\hline & & & & & $n=167$ & 90 to 180 days, $S R M=1.11$ \\
\hline & \multirow[t]{6}{*}{ SFBBS } & \multirow{6}{*}{$\begin{array}{l}\text { Chou } \\
2006 \text { [20] }\end{array}$} & \multirow[t]{6}{*}{ Hospital inpatients } & \multirow[t]{6}{*}{14 days post stroke } & $\mathrm{n}=81$ & 14 to 90 days. \\
\hline & & & & & & 14-item BBS, ES = 0.85 \\
\hline & & & & & & 7-item BBS, ES $=0.78$ \\
\hline & & & & & & 6-item BBS, ES = 0.78 \\
\hline & & & & & & 5-item BBS, ES $=0.70$ \\
\hline & & & & & & 4-item BBS, ES = 0.69 \\
\hline & FM-B & Mao 2002 [17] & Hospital inpatients & 14 days post stroke & $\mathrm{n}=110$ & 14 to 30 days, ES = 0.82 \\
\hline & & & & & $n=93$ & 30 to 90 days, $\mathrm{ES}=0.63$ \\
\hline & & & & & $\mathrm{n}=80$ & 90 to 180 days, $E S=0.33$ \\
\hline & & & & & $\mathrm{n}=93$ & 14 to 90 days, $E S=1.06$ \\
\hline & & & & & $\mathrm{n}=80$ & 14 to 180 days, $E S=1.14$ \\
\hline & SBM & Chien & Inpatient & $<3$ months (mean 41.1 & $n=40$ & 2wks, \\
\hline & & $200 /[21]$ & rehabilitation & $+/-1 / .6)$ & & Equilibrium score, ES $=0.63$ \\
\hline & & & & & & Limits of stability time, $E S=0.27$ \\
\hline & & & & & & Limits of stability path, $\mathrm{ES}=0.33$ \\
\hline & & & & & & Weight shifting, ES $=0.04-0.29$ \\
\hline
\end{tabular}


Table 1 Summary of studies investigating the responsiveness of physical performance measures during inpatient care after stroke, $\mathbf{n}=\mathbf{2 1}$ (Continued)

\begin{tabular}{|c|c|c|c|c|c|c|}
\hline \multirow[t]{14}{*}{ Walking } & \multirow[t]{3}{*}{$10 \mathrm{mWT}$} & $\begin{array}{l}\text { Goldie } \\
1996[22]\end{array}$ & $\begin{array}{l}\text { Inpatient } \\
\text { rehabilitation }\end{array}$ & $\begin{array}{l}\text { Start of rehabilitation, median } \\
16.5 \text { days of hospitalization }\end{array}$ & $n=42$ & $8 w k s, E S=1.17$ \\
\hline & & \multirow[t]{2}{*}{$\begin{array}{l}\text { Salbach } \\
2001[16]\end{array}$} & \multirow[t]{2}{*}{ Acute care hospital } & \multirow[t]{2}{*}{$\begin{array}{l}<8 \text { days (as soon as } \\
\text { ambulatory) }\end{array}$} & \multirow[t]{2}{*}{$n=50$} & $\begin{array}{l}\text { 4wks, Comfortable pace, } E S=0.74, \\
S R M=0.92\end{array}$ \\
\hline & & & & & & $\begin{array}{l}\text { 4Wks, Maximum pace, } E S=0.55 \\
\mathrm{SRM}=0.83\end{array}$ \\
\hline & \multirow[t]{3}{*}{$5 \mathrm{mWT}$} & \multirow[t]{2}{*}{$\begin{array}{l}\text { Salbach } \\
2001[16]\end{array}$} & \multirow[t]{2}{*}{ Acute care hospital } & \multirow[t]{2}{*}{$\begin{array}{l}<8 \text { days (as soon as } \\
\text { ambulatory) }\end{array}$} & \multirow[t]{2}{*}{$\mathrm{n}=50$} & $\begin{array}{l}\text { 4wks, Comfortable pace, } \mathrm{ES}=0.83, \\
\mathrm{SRM}=1.22\end{array}$ \\
\hline & & & & & & $\begin{array}{l}\text { 4Wks, Maximum pace, } E S=0.66 \\
S R M=1.00\end{array}$ \\
\hline & & $\begin{array}{l}\text { English } \\
2006[11]\end{array}$ & $\begin{array}{l}\text { Inpatient } \\
\text { rehabilitation }\end{array}$ & Within 1 wk of admission & $n=61$ & $\begin{array}{l}\text { Within } 1 \text { wk of discharge (mean } \\
\text { time admission to discharge } \\
56.4+/-38.1 \text { days), } E S=0.81\end{array}$ \\
\hline & $2 \mathrm{MWT}$ & $\begin{array}{l}\text { Kosak } \\
2005[23]\end{array}$ & $\begin{array}{l}\text { Inpatient } \\
\text { rehabilitation }\end{array}$ & 28 days (+/- 34 days) & $\mathrm{n}=18$ & 4 wks, SRM=1.34 \\
\hline & 6MWT & $\begin{array}{l}\text { Kosak } \\
2005[23]\end{array}$ & $\begin{array}{l}\text { Inpatient } \\
\text { rehabilitation }\end{array}$ & 28 days (+/- 34 days) & $n=18$ & 4 wks, SRM $=1.52$ \\
\hline & $12 \mathrm{MWT}$ & $\begin{array}{l}\text { Kosak } \\
2005[23]\end{array}$ & $\begin{array}{l}\text { Inpatient } \\
\text { rehabilitation }\end{array}$ & 28 days (+/- 34 days) & $\mathrm{n}=18$ & $4 \mathrm{wks}, \mathrm{SRM}=1.90$ \\
\hline & \multirow[t]{5}{*}{ FAC } & \multirow[t]{2}{*}{$\begin{array}{l}\text { Kollen } \\
2006[24]\end{array}$} & \multirow[t]{2}{*}{ Hospital inpatients } & \multirow[t]{2}{*}{$\begin{array}{l}<14 \text { days (mean days post } \\
\text { stroke }=8.2(\mathrm{SD} 2.8)\end{array}$} & \multirow[t]{2}{*}{$n=101$} & $\begin{array}{l}\text { 4-26wks (weekly in weeks } 1-10 \text {, } \\
\text { fortnightly in weeks 10-20, } \\
\text { once at week 26). }\end{array}$ \\
\hline & & & & & & $\begin{array}{l}\text { Responsiveness ratios based on a } \\
10 \% \text { MCID exceeded the smallest } \\
\text { detectable difference } \\
\text { and ranged from } 4.36 \text { to } 17.70 \text {. }\end{array}$ \\
\hline & & \multirow{3}{*}{$\begin{array}{l}\text { Mehrholz } \\
2007[25]\end{array}$} & \multirow{3}{*}{$\begin{array}{l}\text { Early rehabilitation } \\
\text { centre }\end{array}$} & \multirow[t]{3}{*}{ 30-60 days } & \multirow[t]{3}{*}{$n=55$} & $2 \mathrm{wks} S R M=1.016$ \\
\hline & & & & & & $2 w k s$ to 4 wks SRM $=0.842$ \\
\hline & & & & & & 4 wks to 6 mths SRM $=0.699$ \\
\hline \multirow[t]{14}{*}{ Multiple } & $\begin{array}{l}\text { Modified } \\
\text { EFAM }\end{array}$ & $\begin{array}{l}\text { Liaw } \\
2005[26]\end{array}$ & $\begin{array}{l}\text { Subacute stroke } \\
\text { inpatients }\end{array}$ & Admission & $n=40$ & Discharge, $\mathrm{SRM}=1.1$ \\
\hline & \multirow[t]{6}{*}{ MAS } & \multirow[t]{6}{*}{$\begin{array}{l}\text { English } \\
2006[11]\end{array}$} & \multirow[t]{6}{*}{$\begin{array}{l}\text { Inpatient } \\
\text { rehabilitation }\end{array}$} & \multirow[t]{6}{*}{ Within 1 wk of admission } & \multirow[t]{6}{*}{$n=61$} & $\begin{array}{l}\text { Within } 1 \text { wk of discharge (mean } \\
\text { time admission to discharge } \\
56.4+/-38.1 \text { days). }\end{array}$ \\
\hline & & & & & & Item 1, ES = 1.03 \\
\hline & & & & & & Item 2, ES $=0.74$ \\
\hline & & & & & & Item 3, ES = 0.61 \\
\hline & & & & & & Item 4, ES = 0.85 \\
\hline & & & & & & Item 5, ES = 1.02 \\
\hline & \multirow[t]{3}{*}{$\mathrm{RMA}$} & \multirow{3}{*}{$\begin{array}{l}\text { Kurtais } \\
2009[12]\end{array}$} & \multirow{3}{*}{$\begin{array}{l}\text { Inpatient } \\
\text { rehabilitation }\end{array}$} & \multirow{3}{*}{$\begin{array}{l}\text { Median } 2 \text { months (mean 5.6, } \\
\text { SD 11.2, range } 0.5 \text { to } 78 \text { months) }\end{array}$} & \multirow{3}{*}{$n=107$} & Discharge. \\
\hline & & & & & & $\begin{array}{l}\text { RMA - gross function, } E S=0.51 \\
S R M=0.83\end{array}$ \\
\hline & & & & & & $\begin{array}{l}\text { RMA - leg and trunk, ES }=0.45 \\
\text { SRM }=0.86\end{array}$ \\
\hline & \multirow[t]{4}{*}{$\mathrm{RMI}$} & Hsueh & Hospital inpatients & 14 days & $n=43-$ & 30 days, $S R M=1.14$ \\
\hline & & 2003 [27] & & & & 90 days, $S R M=0.86$ \\
\hline & & & & & & 180 days, SRM $=0.24$ \\
\hline & & $\begin{array}{l}\text { Hsieh } \\
2000 \text { [13] }\end{array}$ & $\begin{array}{l}\text { Inpatient } \\
\text { rehabilitation }\end{array}$ & $\begin{array}{l}\text { Admission to rehabilitation, } \\
\text { median } 24 \text { days post stroke } \\
\text { (range } 7 \text { - } 53 \text { days) }\end{array}$ & $n=38$ & Discharge, $E S=1.28$ \\
\hline
\end{tabular}


Table 1 Summary of studies investigating the responsiveness of physical performance measures during inpatient care after stroke, $\mathbf{n}=\mathbf{2 1}$ (Continued)

\begin{tabular}{|c|c|c|c|c|c|c|}
\hline \multirow[t]{21}{*}{ Multiple } & & $\begin{array}{l}\text { Franchignoli } 2003 \\
{[28]}\end{array}$ & $\begin{array}{l}\text { Inpatient } \\
\text { rehabilitation }\end{array}$ & Admission & $n=73$ & 5 wks, ES $=0.89$ \\
\hline & \multirow[t]{4}{*}{ MRMI } & \multirow{3}{*}{$\begin{array}{l}\text { Hsueh } \\
2003 \text { [27] }\end{array}$} & \multirow[t]{3}{*}{ Hospital inpatients } & \multirow[t]{3}{*}{14 days } & \multirow{3}{*}{$\begin{array}{l}n=43- \\
51\end{array}$} & 30 days, SRM=1.31 \\
\hline & & & & & & 90 days, $\mathrm{SRM}=0.83$ \\
\hline & & & & & & 180 days, $S R M=0.20$ \\
\hline & & $\begin{array}{l}\text { Lennon } \\
2000[29]\end{array}$ & $\begin{array}{l}\text { Inpatient } \\
\text { rehabilitation }\end{array}$ & Admission & $n=16$ & Discharge, ES=1.15 \\
\hline & \multirow[t]{9}{*}{ PASS } & \multirow{3}{*}{$\begin{array}{l}\text { Wang } \\
2004 \text { [19] }\end{array}$} & \multirow[t]{3}{*}{ Hospital inpatients } & \multirow[t]{3}{*}{14 days post stroke } & $n=202$ & 14 to 30 days, SRM $=0.84$ \\
\hline & & & & & $n=167$ & 30 to 90 days, SRM $=0.65$ \\
\hline & & & & & $\mathrm{n}=167$ & 90 to 180 days, $S R M=1.02$ \\
\hline & & \multirow{5}{*}{$\begin{array}{l}\text { Mao } \\
2002[17]\end{array}$} & \multirow[t]{5}{*}{ Hospital inpatients } & \multirow[t]{5}{*}{14 days post stroke } & $n=110$ & 14 to 30 days, ES $=0.89$ \\
\hline & & & & & $n=93$ & 30 to 90 days, $E S=0.64$ \\
\hline & & & & & $n=80$ & 90 to 180 days, $\mathrm{ES}=0.31$ \\
\hline & & & & & $n=93$ & 14 to 90 days, $E S=1.07$ \\
\hline & & & & & $n=80$ & 14 to 180 days, $E S=1.12$ \\
\hline & & $\begin{array}{l}\text { Chien } \\
2007 \text { [21] }\end{array}$ & $\begin{array}{l}\text { Inpatient } \\
\text { rehabilitation }\end{array}$ & $\begin{array}{l}<3 \text { months (mean } 41.1 \\
\text { days }+/-17.6 \text { ) }\end{array}$ & $\mathrm{n}=40$ & $2 w k s, E S=0.41$ \\
\hline & \multirow[t]{3}{*}{ PASS-3P } & \multirow[t]{3}{*}{ Wang 2004 [19] } & \multirow[t]{3}{*}{ Hospital inpatients } & \multirow[t]{3}{*}{14 days post stroke } & $n=202$ & 14 to 30 days, SRM $=0.86$ \\
\hline & & & & & $n=167$ & 30 to 90 days, SRM $=0.67$ \\
\hline & & & & & $n=167$ & 90 to 180 days, $\mathrm{SRM}=1.04$ \\
\hline & 6 SFPASS & $\begin{array}{l}\text { Chien } \\
2007 \text { [30] }\end{array}$ & Hospital inpatients & 14 days post stroke & $n=262$ & 30 days, $E S=0.43-0.44$ \\
\hline & \multirow[t]{3}{*}{ PASS-TC } & \multirow{3}{*}{$\begin{array}{l}\text { Wang } \\
2005[31]\end{array}$} & \multirow[t]{3}{*}{ Hospital inpatients } & \multirow[t]{3}{*}{14 days post stroke } & $n=246$ & 14 to 30 days, SRM $=0.65$ \\
\hline & & & & & $n=203$ & 30 to 90 days, SRM $=0.42$ \\
\hline & & & & & $\mathrm{n}=189$ & 90 to 180 days, $S R M=0.02$ \\
\hline
\end{tabular}

Abbreviations: $E S=$ Effect Size, $S R M=$ Standardized response mean, wks = weeks, $B B S-3 P=$ Berg Balance Scale three point, $B B S=$ Berg Balance Scale, $S F B B S=$ Short Form Berg Balance Scale, $F M-B=$ balance subscale of the Fugl-Meyer test, $S B M=$ Smart Balance Master, $10 \mathrm{mWT}=$ ten metre walk test, $5 \mathrm{mWT}=$ five metre walk test, $2 M W T=$ two minute walk test, $6 M W T=$ six minute walk test, $12 M W T=$ twelve minute walk test, $F A C=$ Functional Ambulation Category, $E F A M=$ Emory Functional Ambulation Profile, MAS = Motor Assessment Scale, RMA = Rivermead Motor Assessment, $R M I=$ Rivermead Mobility Index, MRMI = Modified Rivermead Mobility Index, Motor-FIM = Motor component of the Functional Independence Measure, PASS = Postural Assessment Scale for Stroke Patients, 6 SFPASS = 6-item Short Form Postural Assessment Scale for Stroke Patients, PASS-3P = Three Point Postural Assessment Scale for Stroke Patients, PASS-TC $=$ Postural Assessment Scale for Stroke Patients Trunk Control, $P C B S=$ Postural Control and Balance for Stroke.

\section{Discussion and conclusion}

This systematic review summarised current studies relating to responsiveness of lower limb physical performance measures after stroke. This review demonstrated the variability in the responsiveness of these measures. Within the first four weeks after stroke the measures achieving an ES or SRM of greater than one were the Berg Balance Scale [16], 5-metre walk test [16], 2, 6 and 12 min walking tests [23], the Functional Ambulation Category [25] and the modified Rivermead Mobility Index [27]. When the follow up period was longer, for example more than three months after stroke, the measures achieving an ES or SRM of greater than one were the Berg Balance Scale $[17,18]$ (including modified versions $[17,19])$ and the Postural Assessment Scale for Stroke [17,19] (including a modified version [19]).

This review confirms that responsiveness is specific to the population being investigated and the timeframe of measurement [4]. For example, the Effect Size for the tenmetre walk test $(10 \mathrm{mWT})$ varied from moderate $(\mathrm{ES}=0.55$ to 0.74 ) in one study conducted in an acute hospital with a measurement period of four weeks, to large $(E S=1.17)$ in another study conducted in rehabilitation with a measurement period of eight weeks. This makes identification of responsive measurement tools more challenging, as it is often not appropriate to compare results across studies.

The review was designed to assist clinicians and researchers to select the most appropriate measurement tool for use in their setting or trial. When making this decision a number of factors need to be considered. Firstly, they need to identify studies with a similar setting to their own in Table 1 of the review. For example will the measurement tool be used in an acute ward or rehabilitation unit? Secondly, when after stroke will they first measure the stroke survivors' performance? Table 1 includes the timeframe when studies reported the initial measurement. Thirdly, they need to consider when they plan to re-measure the stroke survivors' performance and find studies in Table 1 of the review that have assessed responsiveness with similar re-measurement 
periods. It is important to recognise that the responsiveness of the same measure can vary greatly if measured two weeks or six months later.

To our knowledge this is the first systematic review to focus on responsiveness of measurement tools in the stroke population. There have been several other reviews of mobility measurement tools in general neurological populations. These reviews found responsiveness to be rarely investigated [5,7]. The mobility measures found to be responsive for use in general neurological populations were the $5 \mathrm{~m}$ and $10 \mathrm{~m}$ walking tests, the $6 \mathrm{MWT}$, the BBS and the Rivermead Mobility Index [5,7]. These measures were also included in our review and shown to be responsive in a stroke specific population.

The results of our systematic review need to be interpreted with caution due to the limitations of the included studies. For example, the two, six and 12-min walk tests demonstrate large Effect Sizes, however were investigated in a small study of just 18 participants [23]. Perhaps if they were investigated in a larger cohort the results would be different. In the review there were nine other studies with sample sizes containing less than 50 participants [10,13,14,16,21-23,26,27,29].

Moreover, a number of studies included in the review included data from the same cohort of stroke survivors $[17,19,20,27,30,31]$. As described above, responsiveness is specific to the sample being investigated. Consequently, it may appear in this review that more samples of stroke survivors have been assessed than is actually the case. Two studies in the review used a sample of convenience and not consecutive stroke survivors [15,23]. This study design may contain significant bias as subjects may be chosen as they are determined to be likely to respond to treatment.

In conclusion, this review has demonstrated the responsiveness of lower limb physical performance measures. The responsiveness of these measures was generally large. However, further systematic investigation of the responsiveness of measures in larger prospective cohort studies is required.

\section{Competing interests}

The authors declare no competing interests.

\section{Authors' contributions \\ $\mathrm{KSCr}$ participated in the study design, carried out the electronic search, extracted data from trials for the review and drafted the manuscript. CS initiated the study design and participated in manuscript editing and writing. KSch participated in the study design, extracted data from trials for the review and participated in manuscript editing and writing. All authors read and approved the final manuscript.}

Received: 28 July 2012 Accepted: 3 January 2013

Published: 10 January 2013

\section{References}

1. Kwakkel G: Extended editorial: towards integrative neurorehabilitation science. Physiother Res Int 2009, 14(3):137-146.
2. Kane RL, Radosevich DM: Conducting Health Outcomes Research. Sudbury, Massachusetts: Jones \& Bartlett Learning; 2011.

3. Mokkink LB, Terwee CB, Patrick DL, Alonso J, Stratford PW, Knol DL, Bouter LM, de Vet HCW: International consensus on taxonomy, terminology, and definitions of measurement properties for health-related patientreported ouctomes: results of the COSMIN study. J Clin Epidemiol 2010, 63:737-745

4. Husted JA, Cook RJ, Farewell VT, Gladman DD: Methods for assessing responsiveness: a critical review and recommendations. J Clin Epidemiol 2000, 53(5):459-468.

5. Tyson S, Connell L: The psychometric properties and clinical utility of measures of walking and mobility in neurological conditions: a systematic review. Clin Rehabil 2009, 23(11):1018-1033.

6. Moher D, Liberati A, Tetzlaff J, Altman DG: Preferred reporting items for systematic reviews and meta-analyses: the PRISMA statement. PLoS Med 2009, 6(7):e1000097. doi:10.1371/journal.pmed.1000097.

7. Salter K, Jutai JW, Teasell R, Foley NC, Bitensky J, Bayley M: Issues for selection of outcome measures in stroke rehabilitation: ICF activity. Disabil Rehabil 2005, 27(6):315-340.

8. Mokkink L, Terwee C, Patrick D, Alonso J, Stratford P, Knol D, Bouter L, de Vet $\mathrm{H}$ : The COSMIN checklist for assessing the methodological quality of studies on measurement properties of health status measurement instruments: an international Delphi study. Qual Life Res 2010, 19(4):539-549.

9. Blum L, Korner-Bitensky N: Usefulness of the Berg Balance Scale in stroke rehabilitation: a systematic review. Phys Ther 2008, 88(5):559-566.

10. Stevenson TJ: Detecting change in patients with stroke using the Berg Balance Scale. Aust J Physiother 2001, 47:29-38.

11. English CK, Hillier SL, Stiller K, Warden-Flood A: The sensitivity of three commonly used outcome measures to detect change amongst patients receiving inpatient rehabilitation following stroke. Clin Rehabil 2006, 20:52-55.

12. Kurtais Y, Kucukdeveci A, Elhan A, Yilmaz A, Kalli T, Tur BS, Tennant A: Psychometric properties of the rivermead motor assessment: its utility in stroke. J Rehabil Med 2009, 41:1055-1061.

13. Hsieh $\mathrm{CL}$, Hsueh IP, Mao HF: Validity and responsiveness of the rivermead mobility index in stroke patients. Scand J Rehabil Med 2000, 32(3):140-142.

14. Katz-Leurer M, Fisher I, Neeb M, Schwartz I, Carmeli E: Reliability and validity of the modified functional reach test at the sub-acute stage post-stroke. Disabil Rehabil 2009, 31(3):243-248.

15. Pyoria O, Talvitie U, Villberg J: The reliability, distribution, and responsiveness of the postural control and balance for stroke test. Arch Phys Med Rehabil 2005, 86:296-302.

16. Salbach NM, Mayo NE, Higgins J, Ahmed S, Finch LE, Richards CL: Responsiveness and predictability of gait speed and other disability measures in acute stroke. Arch Phys Med Rehabil 2001, 82(9):1204-1212.

17. Mao HF, Hsueh IP, Tang PF, Sheu CF, Hsieh CL: Analysis and comparison of the psychometric properties of three balance measures for stroke patients. Stroke 2002, 33:1022-1027.

18. Wood-Dauphinee S, Berg K, Bravo G, Williams Jl: The Balance Scale: responsiveness to clinically meaningful changes. Can J Rehabil 1996, 10(1):35-50

19. Wang CH, Hsueh IP, Sheu CF, Yeo G, Hsieh CL: Psychometric properties of 2 simplified 3-level balance scales used for patients with stroke. Phys Ther 2004, 84(5):430-438.

20. Chou CY, Chien CW, Hsueh IP, Sheu CF, Wang CH, Hsieh CL: Developing a short form of the berg balance scale for people with stroke. Phys Ther 2006, 86:195-204.

21. Chien CW, Hu MH, Tang PF, Sheu CF, Hsieh CL: A comparison of psychometric properties of the smart balance master system and the postural assessment scale for stroke in people who have had mild stroke. Arch Phys Med Rehabil 2007, 88:374-380.

22. Goldie PA, Matyas TA, Evans OM: Deficit and change in gait velocity during rehabilitation after stroke. Arch Phys Med Rehabil 1996, 77(10):1074-1082.

23. Kosak M, Smith T: Comparison of the 2-, 6-, and 12-min walk tests in patients with stroke. J Rehabil Res Dev 2005, 42(1):103-108.

24. Kollen B, van de Port I, Lindeman E, Twisk J, Kwakkel G: Predicting improvement in gait after stroke: a longitudinal prospective study. Stroke 2005, 36:2676-2680. 
25. Mehrholz J, Wagner K, Rutte K, Meissner D, Pohl M: Predictive validity and responsiveness of the functional ambulation category in hemiparetic patients after stroke. Arch Phys Med Rehabil 2007, 88(10):1314-1319.

26. Liaw LJ, Hsieh CL, Lo SK, Lee S, Lin JH: Psychometric properties of the modified Emory functional ambulation profile in stroke patients. Clin Rehabil 2006, 20:429-437.

27. Hsueh IP, Wang CH, Sheu CF, Hsieh CL: Comparison of psychometric properties of three mobility measures for patients with stroke. Stroke 2003, 34(7):1741-1745.

28. Franchignoni F, Tesio L, Benevolo E, Ottonello M: Psychometric properties of the rivermead mobility index in Italian stroke rehabilitation inpatients. Clin Rehabil 2003, 17:273-282.

29. Lennon S, Johnson L: The modified rivermead mobility index: validity and reliability. Disabil Rehabil 2000, 22(18):833-839.

30. Chien CW, Lin JH, Wang CH, Sheu CF, Hsieh CL: Developing a short form of the postural assessment scale for people with stroke. Neurorehabil Neural Repair 2007, 21:81-90.

31. Wang CH, Hsueh IP, Sheu CF, Hsieh CL: Discriminative, predictive, and evaluative properties of a trunk control measure in patients with stroke. Phys Ther 2005, 85(9):887-894.

32. Hsueh IP, Lin JH, Jeng JS, Hsieh CL: Comparison of the psychometric characteristics of the functional independence measure, 5 item Barthel index, and 10 item Barthel index in patients with stroke. $J$ Neurol Neurosurg Psychiatry 2002, 73(2):188-190.

33. Hsieh YW, Hsueh IP, Chou YT, Sheu CF, Hsieh CL, Kwakkel G: Development and validation of a short form of the Fugl-Meyer motor scale in patients with stroke. Stroke 2007, 38:3052-3054.

doi:10.1186/1471-2377-13-4

Cite this article as: Scrivener et al: A systematic review of the responsiveness of lower limb physical performance measures in inpatient care after stroke. BMC Neurology 2013 13:4.

\section{Submit your next manuscript to BioMed Central and take full advantage of:}

- Convenient online submission

- Thorough peer review

- No space constraints or color figure charges

- Immediate publication on acceptance

- Inclusion in PubMed, CAS, Scopus and Google Scholar

- Research which is freely available for redistribution 